\title{
Implementation of a short-tip tapping-mode tuning fork near-field scanning optical microscope
}

\author{
N. H. LU*, C. W. HUANG†, C. Y. CHEN†, C. F. YU §, T. S. KAO†, \\ Y. H. FU† \& D. P. TSAI† \\ *Department of Electronic Engineering, De Lin Institute of Technology, Tuchen, Taipei County, Taiwan, \\ 23646, R.O.C. \\ †Department of Physics, National Taiwan University, Taipei, Taiwan, R.O.C. \\ $\ddagger$ Department of Physics, National Taiwan Normal University, Taipei, Taiwan, R.O.C. \\ §Institute of Optoelectronic Sciences, National Taiwan Ocean University, Keelung, Taiwan, R.O.C.
}

Key words. Near-field scanning optical microscopy, short tip, tapping-mode, tuning fork.

\section{Summary}

We present the implementation of a short-tip tapping-mode tuning fork near-field scanning optical microscope. Tapping frequency dependences of the piezoelectric signal amplitudes for a bare tuning fork fixed on the ceramic plate, a short-tip tapping-mode tuning fork scheme and an ordinary tappingmode tuning fork configuration with an 80-cm optical fibre attached are demonstrated and compared. Our experimental results show that this new short-tip tapping-mode tuning fork scheme provides a stable and high $Q$ factor at the tapping frequency of the tuning fork and will be very helpful when long optical fibre probes have to be used in an experiment. Both collection and excitation modes of short-tip tapping-mode tuning fork near-field scanning optical microscope are applied to study the near-field optical properties of a single-mode telecommunication optical fibre and a green InGaN/GaN multiquantum well light-emitting diode.

\section{Introduction}

Advances in nanoscience and nanotechnology advocate rapid progress in new techniques and instruments which have the ability to perform nanoscale fabrication or to offer high spatial resolution characterization down to the nanometre dimension. Owing to its versatile applications in nanostructure fabrication (Massanell et al., 1996; Herndon et al., 1999), high density optical (Tsai \& Lin, 2000) and magneto-optical (Fumagalli et al., 1998) data storage analysis, and diagnostics of low-dimensional semiconductor heterostructures (Eytan et al., 1998; Eah etal., 2002), near-field scanning optical

Correspondence: N. H. Lu. Tel.: +8862 2273 4415; fax: +88622261 1492; e-mail:nhlu@sitc.edu.tw microscopy (NSOM) has proven a powerful tool in nanometric science and technology.

NSOM roughly falls into two categories, depending on the functionality of the optical fibre probe. In collection mode NSOM, the fibre probe detects the local signal coupled to the tip aperture. In excitation mode NSOM, instead of acting as a local detector, the fibre probe serves as a nano-illuminator. By bringing the tapered fibre probe into close proximity with the sample surface, the inevitable diffraction limit encountered in conventional far-field optical methods can be overcome.

Since initial demonstrations that crystal quartz tuning forks can be used as sensors for acoustic (Güthner et al., 1989) and force (Karrai \& Grober, 1995) microscopy, applications of this technique have been extended to near-field optical microscopy (Davydov et al., 1999; Crottini et al., 2002), atomic force microscopy (Giessibl, 2000; Callaghan et al., 2002), and electrostatic force microscopy (Seo et al., 2002; Wang et al., 2002). Recently, we demonstrated the working principles of the tapping-mode tuning fork near-field scanning optical microscopy (TMTF-NSOM; Tsai \& Lu, 1998) and successfully applied this new method to study the physical properties of a single mode optical fibre waveguide (Tsai et al., 1999), superresolution near-field structures (Tsai \& Lin, 2000), and semiconductor lasers (Lu et al., 2001).

In contrast to conventional optical shear-force mode NSOM, the distance regulation between the sample surface and the cusp of the fibre probe is realized by a non-optical and normal-force feedback control method in TMTF-NSOM. Consequently, TMTF-NSOM saves the trouble resulting from elaborate optical beam alignment and interference between the near-field optical signal and the force sensing optics, and provides all the advantages afforded by the tapping-mode atomic force microscopy, including high spatial resolution, high sensitivity and excellent stability. However, in some cases, the 
friction or the massive load could be a critical issue. For example, when an optical fibre longer than $80 \mathrm{~cm}$ was used, we could hardly sense any appreciable induced voltage difference between the two electrodes of the tuning fork under excitation. As a result, neither any significant feature of resonance response nor any noticeable change in the feedback signal as the tip approaches the sample surface could be observed. Here, we present a new scheme, the short-tip TMTF-NSOM, in which a cleaved short fibre tip attached to one tine of the tuning fork is used as the force sensing element, to overcome the problems mentioned above.

In order to demonstrate the feasibility of this new method, we apply collection and excitation modes of short-tip TMTFNSOM to measure the near-field optical properties of a singlemode telecommunication optical fibre and a green InGaN/ GaN multiquantum well light-emitting diode.

\section{Experimental}

The TMTF-NSOM system is based on a commercial atomic force microscope (Stand Alone, Park Scientific Instruments). The force-sensing element consists of a near-field optical fibre probe attached to a crystal quartz tuning fork. The tapered optical fibre probe was manufactured by pulling a single-mode optical fibre while heating locally with a $\mathrm{CO}_{2}$ laser and subsequently immersing the tip in a saturated solution of ammonium bifluoride $\left(\mathrm{NH}_{4} \mathrm{~F} \cdot \mathrm{HF}\right)$ for a few minutes. The tapers were finally coated on the sides by a thin layer of aluminium to confine the light. Using this procedure, typical aperture sizes with diameters between 50 and $80 \mathrm{~nm}$ can be realized.

In an attempt to overcome problems resulting from massive load as long optical fibres have to be used in the experiment, we devised the short-tip TMTF-NSOM. Figure 1 shows a schematic of the short-tip tapping-mode tuning fork set-up. The optical fibre probe is attached perpendicular to the end of one tine of the tuning fork, while the other tine is glued to a ceramic plate with the fork protruding $\approx 3.5 \mathrm{~mm}$ from the

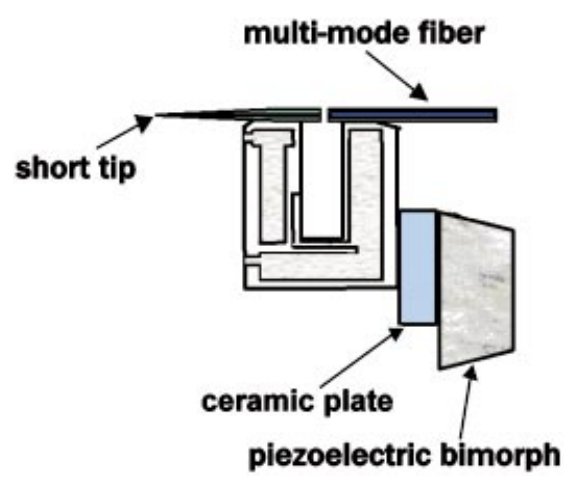

Fig. 1. Schematic construction of the tapping-mode tuning fork with a short fibre probe. The air gap between the end-faces of the short tip and the multimode optical fibre is $\approx 10 \mu \mathrm{m}$ or less. The tuning fork is mechanically excited by the piezoelectric bimorph. edge of the plate. The ceramic plate is tightly clamped onto and mechanically excited by a piezoelectric bimorph. Both the tuning fork and the fibre tip vibrate in a direction normal to the sample surface. The $Q$ factor and the resonance frequency of the tuning fork proper are $\approx 80000$ and $32.768 \mathrm{kHz}$, respectively. The tip protrudes $\approx 1 \mathrm{~mm}$ from the fork edge and the short fibre tip is obtained by cleaving the optical fibre halfway between the two prongs. A flat-ended multimode fibre is then glued to the other prong of the tuning fork with the air gap between the end-faces of the short tip and the multimode optical fibre being $\approx 10 \mu \mathrm{m}$ or less. All these procedures are performed with the aid of a precision multi-axis translation stage and $\mathrm{a} \times 90$ stereo optical microscope. Care and patience must be exercised in aligning the long axis of the short fibre tip with that of the multimode optical fibre for efficient coupling of the light. Typical throughput (i.e. output power from the tip aperture vs. input power through the multimode fibre) of this configuration is $10^{-4}$.

\section{Results and discussion}

Plotted in Fig. 2 are the frequency dependences of the induced voltage differences between the two electrodes of the tuning fork for the following three cases: (a) a bare tuning fork (i.e. without any optical fibre attached) fixed on the ceramic plate, (b) a short-tip TMTF scheme and (c) an ordinary TMTF configuration with an 80-cm optical fibre attached. The $Q$ factors are obtained by dividing the resonance peak frequency by the frequency full-width at half-maximum of the piezoelectric signal amplitude at resonance. As seen in Fig. 2, both the bare tuning fork and the short-tip TMTF show sharp and pronounced resonance peaks at 32.49 and $31.45 \mathrm{kHz}$ with $Q$ factors 192 and 181, respectively, whereas the piezoelectric signal of the ordinary TMTF is rather broad. Indeed, when the optical fibre was longer than $80 \mathrm{~cm}$ in the ordinary TMTF configuration, we could hardly detect any appreciable induced voltage difference between the two electrodes of the tuning fork under excitation. In general, the resonance responses of the bare tuning fork and the short-tip TMTF are similar because of the light mass of the short fibre probe. We also found that the resonance frequency response of the short-tip TMTF is almost independent of the length of the multimode optical fibre.

To evaluate the functional performance of this new method, we performed both collection and excitation modes of shorttip TMTF-NSOM measurements of a single-mode telecommunication optical fibre and a green InGaN/GaN multiquantum well light-emitting diode. Near-field transverse electromagnetic mode of a single-mode telecommunication optical fibre (Prime Optical Fibre Corporation, SMF-1310, operating wavelength at $1.31 \mu \mathrm{m}$ and cut-off wavelength at $1.15 \mu \mathrm{m}$ ) fabricated by vapour axial deposition process was imaged by the collection mode of short-tip TMTF-NSOM. The sample is a step-index germanosilicate fibre, and the nominal diameters 


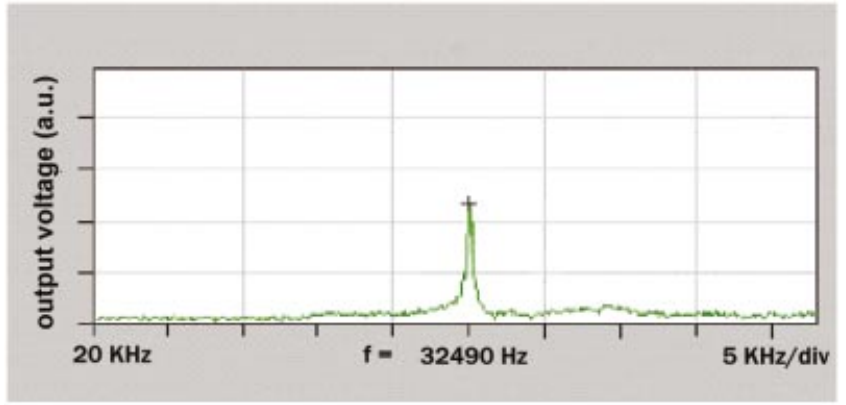

(a)

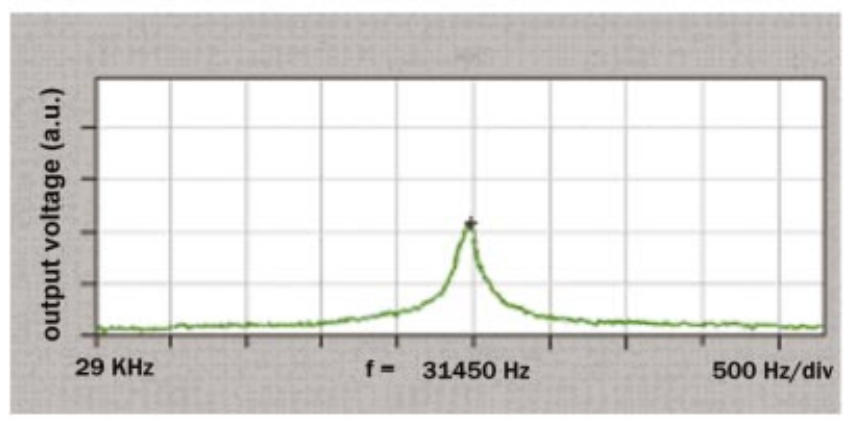

(b)

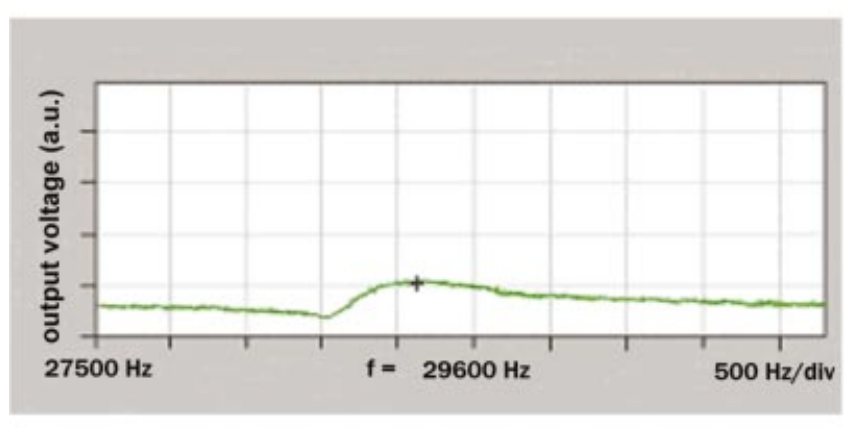

(c)

Fig. 2. Tapping frequency dependences of piezoelectric signal amplitudes for a bare tuning fork (i.e. without any optical fibre attached) fixed on a ceramic plate (a), a short-tip TMTF scheme (b), and an ordinary TMTF configuration with $80 \mathrm{~cm}$ optical fibre (c).

of the core and the cladding are 9 and $125 \mu \mathrm{m}$, respectively. The collection mode is carried out by scanning the probe above one end-face of the optical fibre, whereas the other end of the fibre is coupled by a laser beam. Figure 3(a) shows the near-field intensity distribution of the $\mathrm{LP}_{01}$ mode when the optical fibre is illuminated at the other end-face by a laser diode of emission wavelength $1310 \mathrm{~nm}$. The mode field diameter of the $\mathrm{LP}_{01}$ mode is measured to be $10.0 \mu \mathrm{m}$, which is consistent with the product specifications. When the light source is replaced by a 1550-nm laser diode, the mode field diameter of the $\mathrm{LP}_{01}$ mode turns out to be $11.0 \mu \mathrm{m}$ (not shown).

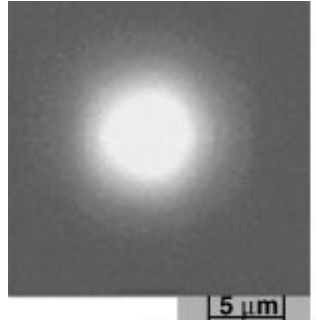

(a)

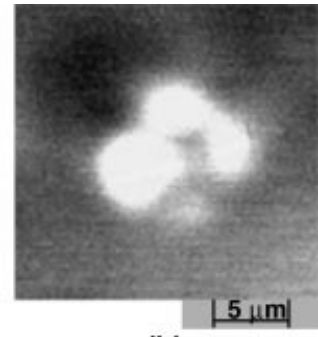

(b)
Fig. 3. Near-field transverse electromagnetic mode pattern measured at the cleaved facet of a single mode telecommunication optical fibre excited by laser wavelengths of $1310 \mathrm{~nm}$ (a) and $633 \mathrm{~nm}$ (b). The scanned area is $20 \times 20 \mu \mathrm{m}^{2}$.

Figure 3(b) shows the cross-sectional near-field output of the single-mode telecommunication optical fibre excited by a 633-nm HeNe laser. As we know, if the propagating wavelength is shorter than the single mode cut-off wavelength, the optical fibre becomes multimoded. The modal pattern indicates that, in this situation, the main higher order guided modes are $\mathrm{LP}_{11}$ and $\mathrm{LP}_{21}$. The asymmetric intensity distribution is attributed to the inevitable fibre bend encountered in the experiment (Syms \& Cozens, 1992).

Excitation mode of short-tip TMTF-NSOM is used to perform the near-field photocurrent (NPC) experiment of an InGaN/ GaN multiquantum well light-emitting diode of emission wavelength $527 \mathrm{~nm}$. The sample studied was fabricated by metal organic chemical vapour deposition. The active region consists of three $25-\AA$ wide $\operatorname{In}_{0.5} \mathrm{Ga}_{0.5} \mathrm{~N}$ quantum wells separated by $80-\AA$ thick $\mathrm{GaN}$ barriers. On top of the active region were grown $300 \AA$ Mg-doped $\mathrm{Al}_{0.12} \mathrm{Ga}_{0.88} \mathrm{~N}$ and $1200 \AA$ $\mathrm{Mg}$-doped GaN epitaxial layers. The p-electrode is obtained by evaporating a $150-\AA ̊$ thick $\mathrm{Ni} / \mathrm{Au}$ film onto the p-type GaN. NPC was generated by scanning a fibre tip on the top surface of the sample. A mechanically chopped 633-nm HeNe laser beam with modulation frequency $f=500 \mathrm{~Hz}$ was coupled to the optical fibre as the pumping source.

Figure 4 represents the simultaneously measured surface morphology (a) and NPC (b) micrographs of the InGaN/GaN quantum well structure. The scanned area is $10 \times 10 \mu^{2}$ and the excitation power density is $3.4 \mathrm{~kW} \mathrm{~cm}^{-2}$. The NPC distribution shows a high contrast region corresponding to the inhomogeneous stripe with a root mean square roughness of $2 \mathrm{~nm}$ appearing in the central part of the topographic image. Because the excitation photon energy $(1.96 \mathrm{eV})$ is smaller than the band gap energy $(2.35 \mathrm{eV})$, the photocurrent is generated by a two-step excitation process via deep energy levels in the gap (Lang \& Henry, 1978; Lu et al., 1999). The topographic information alone suggests that the roughness could be due to non-uniform film growth of $\mathrm{Ni} / \mathrm{Au}$ or misfit dislocations (Jain et al., 2000). However, in combination with the NPC data, the roughness appearing in the central region can be unambiguously attributed to the misfit dislocations. 


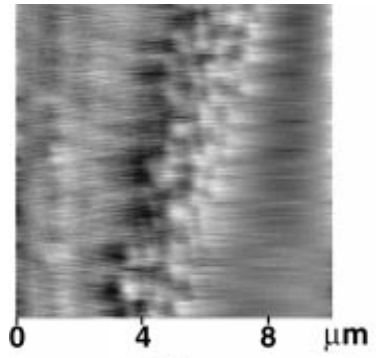

(a)

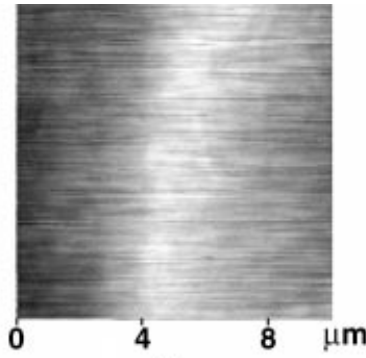

(b)
Fig. 4. Simultaneously measured surface morphology (a) and nearfield photocurrent (b) micrographs of the InGaN/GaN quantum well structure. The excitation power density is $3.4 \mathrm{~kW} \mathrm{~cm}^{-2}$. The near-field photocurrent distribution shows a high contrast region corresponding to the inhomogeneous stripe with a root mean square roughness of $2 \mathrm{~nm}$ appearing in the central part of the topographic image.

However, the inhomogeneous longitudinal stripe observed in the left part of the topographic image does not correspond to any photoresponse. We thus ascribe such inhomogeneities to non-uniform film growth of $\mathrm{Ni} / \mathrm{Au}$.

\section{Conclusions}

In conclusion, we have shown how to implement a short-tip TMTF-NSOM. Our results demonstrate that this new scheme provides a stable and high $Q$ factor at the tapping frequency of the tuning fork and will be very helpful when long optical fibre probes have to be used in an experiment. The collection mode of the short-tip TMTF-NSOM is used to image the near-field transverse electromagnetic mode emanating from the cleaved facet of a single-mode telecommunication opticalfibre. Dominant guided modes inside the optical fibre excited by laser wavelengths of 1310 and $633 \mathrm{~nm}$ can be well resolved by using this technique. Furthermore, the excitation mode of short-tip TMTF-NSOM is applied to perform the sub-band gap excitation NPC experiment of a green InGaN/GaN multiquantum well light-emitting diode. In combination with the NPC data, the spatial inhomogeneities observed in the central and left parts of the topographic micrograph can be unambiguously attributed to misfit dislocations and nonuniform film growth of $\mathrm{Ni} / \mathrm{Au}$, respectively.

\section{Acknowledgements}

This study was supported by the National Science Council of the Republic of China under Grant No. 91-2112-M-237-001. Furthermore, this study was supported in part by MOEA under Grant No. 91-EC-17-A-08-SI-0006.

\section{References}

Callaghan, F.D., Yu, X. \& Mellor, C.J. (2002) Dynamic force microscopy in superfluid helium. Appl. Phys. Lett. 81, 916-918.
Crottini, A., Staehli, J.L., Deveaud, B., Wang, X.L. \& Ogura, M. (2002) Ultra stable tuning fork sensor for low-temperature near-field spectroscopy. Ultramicroscopy, 90, 97-101.

Davydov, D.N., Shelimov, K.B., Haslett, T.L. \& Moskovits, M. (1999) A near-field scanning optical microscope with a high $Q$-factor piezoelectric sensing element. Appl. Phys. Lett. 75, 1796-1798.

Eah, S., Jhe, W. \& Arakawa, Y. (2002) Near-field optical photoluminescence microscopy of high-density InAs/GaAs single quantum dots. Appl. Phys. Lett. 80, 2779-2781.

Eytan, G., Yayon, Y., Rappaport, M., Shtrikman, H. \& Bar-Joseph, I. (1998) Near-field spectroscopy of a gated electron gas: direct evidence for electron localization. Phys. Rev. Lett. 81, 1666-1669.

Fumagalli, P., Rosenberger, A., Eggers, G., Münnemann, A., Held, N. \& Güntherodt, G. (1998) Quantitative determination of the Kerr rotation by scanning near-field magneto-optic microscopy. Appl. Phys. Lett. 72, 2803-2805.

Giessibl, F.J. (2000) Atomic resolution on Si(111)-(7×7) by noncontact atomic force microscopy with a force sensor based on a quartz tuning fork. Appl. Phys. Lett. 76, 1470-1472.

Güthner, P., Fischer, U.C. \& Dransfeld, K. (1989) Scanning near-field acoustic microscopy. Appl. Phys. B, 48, 89-92.

Herndon, M.K., Collins, R.T., Hollingsworth, R.E., Larson, P.R. \& Johnson, M.B. (1999) Near-field scanning optical nanolithography using amorphous silicon photoresists. Appl. Phys. Lett. 74, 141-143.

Jain, S.C., Willander, M., Narayan, J. \& Van Overstraeten, R. (2000) IIInitride: growth, characterization, and properties. J. Appl. Phys. 87, 965-1006.

Karrai, K. \& Grober, R.D. (1995) Piezoelectric tip-sample distance control for near field optical microscopes. Appl. Phys. Lett. 66, 1842-1844.

Lang, D.V. \& Henry, C.H. (1978) Scanning photocurrent microscopy: a new technique to study inhomogeneously distributed recombination centers in semiconductors. Solid-State Electron. 21, 1519-1524.

Lu, N.H., Lin, W.C. \& Tsai, D.P. (2001) Tapping-mode tuning-fork near-field scanning optical microscopy of low power semiconductor lasers. J. Microsc. 202, 172-175.

Lu, N.H., Tsai, D.P., Chang, C.S. \& Tsong, T.T. (1999) Optical characterization of visible multiquantum-well semiconductor lasers by collection/ excitation modes of scanning near-field optical microscopy. Appl. Phys Lett. 74, 2746-2748.

Massanell, J., García, N. \& Zlatkin, A. (1996) Nanowriting on ferroelectric surfaces with a scanning near-field optical microscope. Opt. Lett. 21, $12-14$.

Seo, Y., Jhe, W. \& Hwang, C.S. (2002) Electrostatic force microscopy using a quartz tuning fork. Appl. Phys. Lett. 80, 4324-4326.

Syms, R. \& Cozens, J. (1992) Optical Guided Waves and Devices. McGrawHill, London.

Tsai, D.P. \& Lin, W.C. (2000) Probing the near fields of the super-resolution near-field optical structure. Appl. Phys. Lett. 77, 1413-1415.

Tsai, D.P. \& Lu, Y.Y. (1998) Tapping-mode tuning fork force sensing for near-field scanning optical microscopy. Appl. Phys. Lett. 73, $2724-$ 2726.

Tsai, D.P., Yang, C.W., Lo, S. \& Jackson, H.E. (1999) Imaging local index variations in an optical waveguide using a tapping-mode near-field scanning optical microscope. Appl. Phys. Lett. 75, 1039-1041.

Wang, Z., Bao, J., Zhang, H. \& Guo, W. (2002) High-resolution surface charge image achieved by a multiforce sensor based on a quartz tuning fork in electrostatic force microscope. Appl. Phys. Lett. 81, 13001302. 